\title{
Obtención por vía pulvimetalúrgica de materiales compuestos de matriz de aluminio reforzados con nitruros
}

\author{
J. B. FOGAGNOLO, M. H. ROBERT, J. L. ORTIZ1 ${ }^{1}$ V. AMIGÓ², J. M. TORRALBA ${ }^{3}$ \\ Universidad Estadual de Campinas, Ciudad Universitaria de Campinas, 130283-970, Campinas-SP Brasil. \\ ${ }^{1}$ Instituto Tecnológico y de Estudios Superiores de Monterrey, Campus Querétaro, Jesús Oviedo 10, 76130 Querétaro, Qro. México. \\ ${ }^{2}$ Universidad Politécnica de Valencia, Camino de Vera, 46022 Valencia. \\ ${ }^{3}$ Universidad Carlos III de Madrid, Avd. Universidad 30, 28911 Leganés, Madrid.
}

\begin{abstract}
La utilización de los materiales compuestos de matriz de aluminio como materiales estructurales está en continuo crecimiento principalmente en la industria automovilística y aeroespacial. El desarrollo de nuevos tipos de refuerzos es una de las áreas de mayor interés en las más actuales investigaciones. Este trabajo investiga el uso de nitruros como refuerzo en materiales compuestos, de matriz de aleación AA6061, obtenidos vía pulvimetalúrgica y extrusión, presentando los aspectos microestruturales y las características mecánicas.
\end{abstract}

Palabras clave: Materiales compuestos de matriz metálica, pulvimetalurgia, extrusión.

Obtaining aluminium matriz composities reinforced with nitrides by powder metallurgy

The use of aluminium metal matrix composites as structural materials is increasing continuously, mainly in the automotive and aerospace industries. The development of new kind of reinforcements is one of the areas of greater interest in current researches. This work investigates the use of nitrides as reinforcement for composite materials, with Al 6061 matrix, obtained by powder metallurgy and extrusion, presenting their microstructural features and their mechanical characteristics.

Key words: Metal matrix composites, powder metallurgy, extrusion.

\section{INTRODUCCIÓN}

Los materiales compuestos de matriz metálica reforzados con materiales cerámicos han sido ampliamente estudiados dadas sus excelentes propiedades desde el punto de vista estructural, en la industria automovilística y aerospacial. Gran parte de los trabajos hechos con compuestos de matriz metálica utilizan como matriz aleaciones de aluminio, dado que su punto de fusión es suficientemente alto para satisfacer los requisitos de muchas aplicaciones y suficientemente bajo para posibilitar moderados costes de proceso. Además, su peso específico es bajo y tienen gran capacidad para acomodarse a una amplia variedad de tipos de refuerzos, como $\mathrm{Al}_{2} \mathrm{O}_{3}$ o $\mathrm{SiC}$, en forma de fibras, whiskers o partículas. La técnica pulvimetalúrgica para la obtención de materiales compuestos tiene como principales ventajas una mejor distribución del refuerzo en la matriz metálica, así como un ahorro en materias primas y menor consumo energético en comparación con la metalurgia convencional (1).

Las investigaciones más recientes se centran en el desarrollo de nuevas matrices y refuerzos para materiales compuestos (2), (3). Los compuestos con refuerzos discontinuos, a pesar de tener peor comportamiento a la fractura que con refuerzos en forma de fibras, tienen menores costes de fabricación y propiedades más isótropas.

Este trabajo tiene como objetivo estudiar la viabilidad de añadir nuevos refuerzos en forma de partículas a los materiales compuestos de matriz de aluminio, como son los nitruros, que no se están empleando industrialmente en la actualidad.

\section{PROCEDIMIENTO EXPERIMENTAL}

Como matriz fue utilizada la aleación Al 6061, con tamaño medio de partícula de $75 \mathrm{~mm}$, suministrada por "The Aluminium Powder Co. Ltd.". Como refuerzos, fueron utilizados nitruro de aluminio y nitruro de silicio, con tamaño medio de partícula de 14 y 8,6 $\mu \mathrm{m}$, respectivamente, suministrados por "Advanced Refractory Technologies, Inc.". La Figura 1 presenta la morfología de las materias primas utilizadas y la Tabla I presenta las principales características de los nitruros utilizados como refuerzos.

Para la obtención de los materiales compuestos vía pulvimetalúrgica, se realizaron las siguientes etapas:

a) Mezcla de la matriz con el refuerzo en molino de bolas para la obtención del polvo compuesto (Relación bolas/carga en peso: 10/1; diámetro de las bolas: $10 \mathrm{~mm}$; velocidad: 150 revoluciones por minuto; tiempo de mezcla: 90 minutos).

b) Compactación uniaxial en frío de la mezcla para la obtención de un compacto en verde cilíndrico (presión de $200 \mathrm{MPa}$ ).

c) Extrusión del compacto en verde para la obtención del material compuesto en su forma acabada, sin tratamiento térmico (temperatura de extrusión: $550{ }^{\circ} \mathrm{C}$, presión de extrusión: $400 \mathrm{MPa}$, reducción de área: 25/1).

Tras la extrusión de los materiales, se estudia su microestructura mediante microscopía óptica y electrónica de barrido. Para la determinación de las propiedades mecánicas se realizan ensayos de tracción sobre las muestras extruídas (Norma EN 10.002-1), a una velocidad de $1 \mathrm{~mm} / \mathrm{min}$ a temperatura ambiente. 


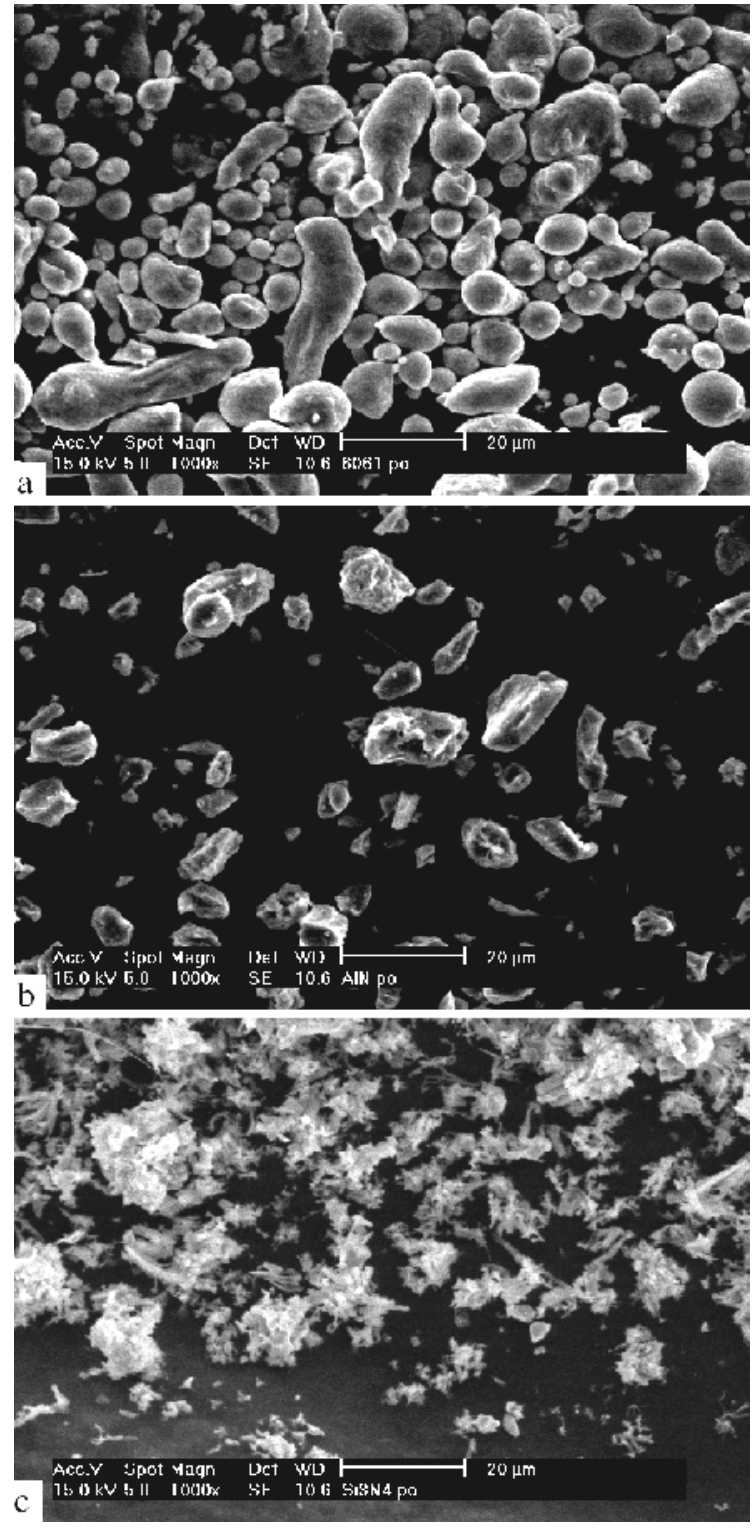

Figura 1: Microestruturas de la aleación AA6061 (a), utilizada como matriz, y del nitruro de aluminio (b) y nitruro de silicio (c), utilizados como refuerzos.

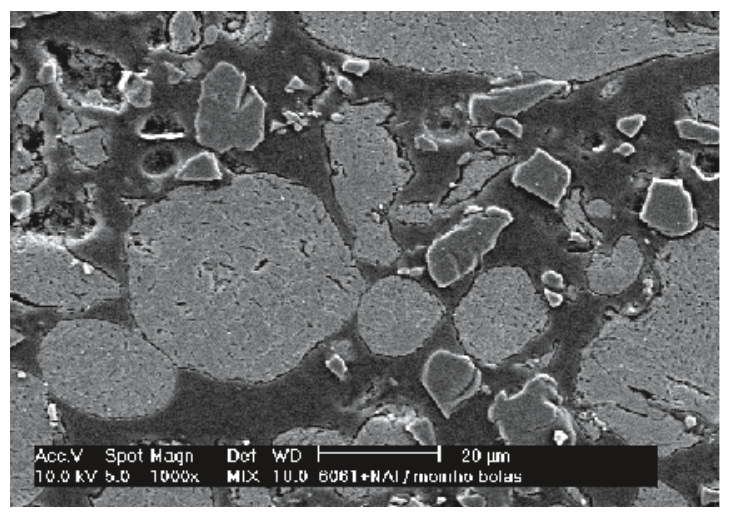

Figura 2: Microestrutura del polvo compuesto reforzado con $15 \%$ de nitruro de aluminio.

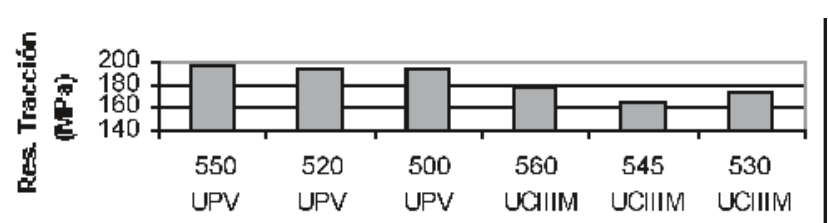

Temperatura de extrusión $\left({ }^{\circ} \mathrm{C}\right) /$ Procedencia de las muestras

Figura 3: Resistencia a tracción de la aleación AA6061 en función de la temperatura de extrusión.

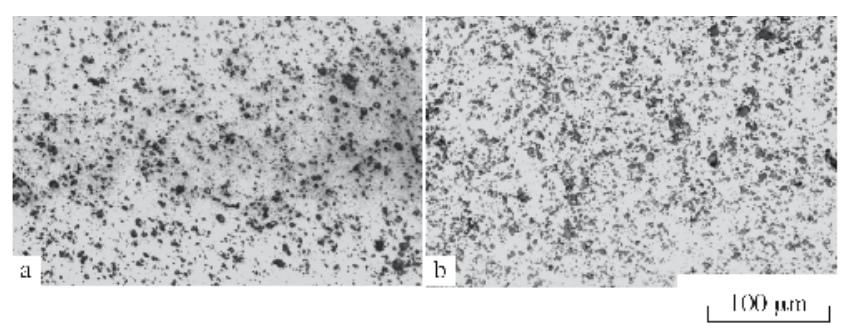

Figura 4: Microestructura del compuesto $\mathrm{Al} 6061$ con (a) 5 y (b) $15 \%$ de nitruro de silicio.

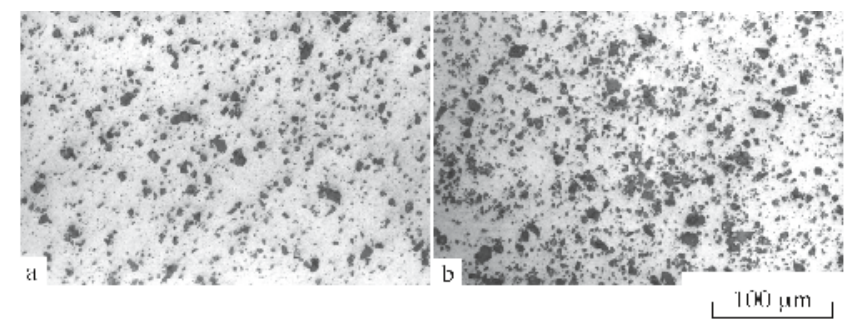

Figura 5: Microestrutura del compuesto Al 6061 con (a) 5 y (b) $15 \%$ de nitruro de aluminio.
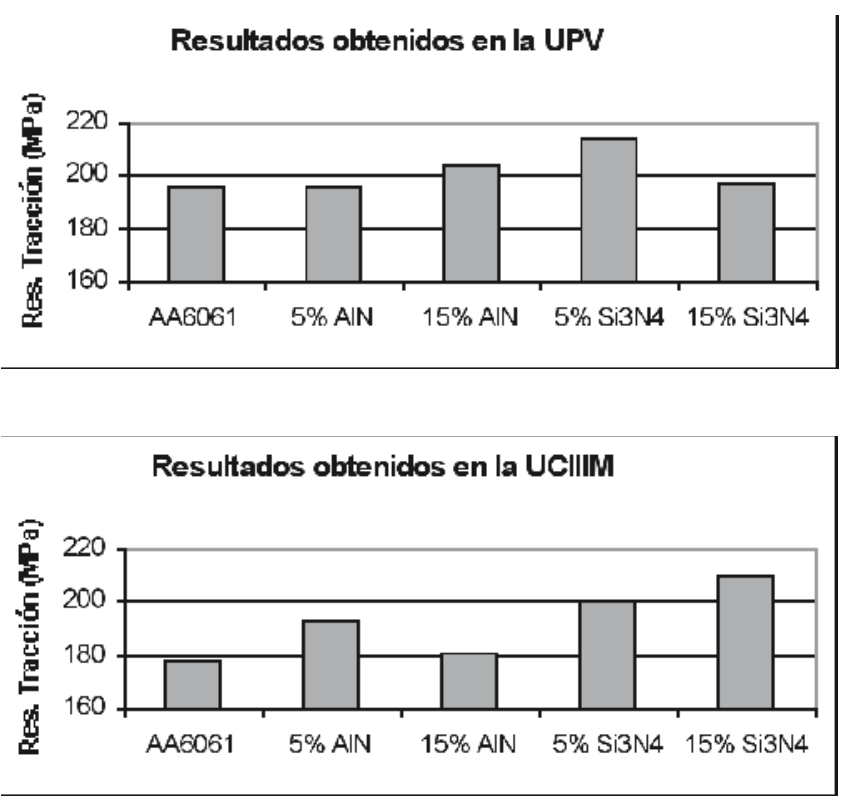

Figura 6: Resistencia a tracción de los compuestos obtenidos. 


\section{RESULTADOS}

Se obtuvieron polvos compuestos con distribución homogénea de refuerzo, indicando que el proceso de mezcla en molino de bolas fue satisfactorio. La Figura 2 presenta la microestructura del polvo compuesto reforzado con $15 \%$ de AIN. Tras la compactación, se obtuvieron compactos con aproximadamente $80 \%$ de la densidad teórica del material.

Para la optimización del proceso de extrusión y también la posterior comparación de propiedades entre los materiales reforzados y sin reforzar, la aleación AA6061 fue extruída a diferentes temperaturas. Se observó que, dentro del intervalo de temperatura investigado $\left(500\right.$ a $\left.560^{\circ} \mathrm{C}\right)$, no se ha encontrado una relación clara entre la temperatura de extrusión y la resistencia a la tracción de la aleación extruída, dentro del intervalo estudiado en cada centro de investigación, como puede verse en la Figura 3. Así, fue elegida la temperatura más alta por ser la temperatura en la cual se necesitan menores presiones de extrusión. Tras la extrusión, se obtuvieron materiales con aproximadamente $98 \%$ de la densidad teórica del material, lo que significa que los materiales habian densificado casi totalmente, siendo la porosidad prácticamente nula. La Figura 4 presenta la microestructura del compuesto reforzado con (a) 5 y (b) $15 \%$ de nitruro de silicio y la Figura 5 presenta la microestructura del compuesto reforzado con (a) 5 y (b) $15 \%$ de nitruro de aluminio. En estas microestructuras sin ataque, es posible apreciar la distribución homogénea del refuerzo en la matriz.

Los ensayos de tracción indican que la resistencia de los materiales compuestos mejora moderadamente en comparación con la aleación sin refuerzo, como puede observarse en la Figura 6. Las razones que llevan a distintos resultados de tracción en ambos centros (Figuras 3 y 6 ) están relacionadas, posiblemente, con la presencia de defectos superficiales en el material extruído. Estos defectos, que sólo pueden eliminarse mediante un mecanizado posterior que no es objeto de la presente investigación, tienen una fuerte influencia sobre el ensayo de tracción y la dispersión de resultados que pueden aparecer en el mismo. Ensayos todavía en curso en las dos
TABLA I: CARACTERÍSTICAS DE LOS MATERIALES EMPLEADOS COMO REFUERZO.

\begin{tabular}{|c|c|c|c|c|}
\hline Material & $\begin{array}{c}\text { Hierro } \\
(\mathbf{p p m})\end{array}$ & $\begin{array}{c}\text { Carbono } \\
(\%)\end{array}$ & $\begin{array}{c}\text { Tamaño Medio } \\
\text { de Partícula }(\mu \mathrm{m})\end{array}$ & $\begin{array}{c}\text { Densidad } \\
\left(\mathrm{g} / \mathbf{c m}^{3}\right)\end{array}$ \\
\hline Nitruro de silicio & 47 & $<0,12$ & 8,6 & 3,44 \\
Nitruro de aluminio & 230 & $<0,1$ & 14 & 3,26 \\
\hline
\end{tabular}

Universidades investigan las propiedades frente al desgaste y a la corrosión, con el fin de lograr una caracterización más completa de los materiales.

\section{CONCLUSIONES}

La temperatura de extrusión, dentro del intervalo de 500 a $560^{\circ} \mathrm{C}$, no influye en la resistencia a tracción de la aleación AA6061 obtenida vía pulvimetalúrgica y extrusión.

La resistencia a tracción en los compuestos de matriz de aluminio AA6061 reforzados con nitruro de silicio y nitruro de aluminio mejora moderadamente en comparación con la aleación sin refuerzo.

\section{BIBLIOGRAFÍA}

1 - H. F. LEE, F. BOEY, K. A. KHOR, M. J. TAN, J. GAN, N. L. LOH, "The Production of Aluminium Alloy Composites Using a Cold Isostatic Press and Extrusion Approach", Journal of Materials Processing Technology, vol. 29, p.245-253. (1992).

2 - M. E. SMAGORINSKI, P. G. TZANTRIZOS, S. GRENIER, A. CAVASIN, T. BRZEZINSKI, G. KIM, "The Properties and Microstructure of Al-based Composites Reinforced with Ceramic Particles", Materials Science and Engineering A, vol. A244, p.86-90. (1998).

3 - J. L. ROSSI, "Reinforcements for Metal Matrix Composites Produced by Powder Metallurgy", Proceedings of the 1998 Powder Metallurgy World Congress and Exhibition, Granada, España, vol 5, p.157-161. (1998). 